\section{Minimal incision posterior approach levator plication for aponeurotic ptosis}

DS Ng, E Chan and ST Ko

\begin{abstract}
Purpose To assess the efficacy and predictability of a minimal incision posterior approach levator plication technique for correction of involutional ptosis.

Method Retrospective chart review of patients with involutional aponeurotic ptosis underwent minimal incision posterior approach levator plication technique between August 2013 and June 2014 by a single surgeon. The upper lid was double everted, and the conjunctiva and Muller's muscle layers were incised vertically until the levator aponeurosis could be identified. The incision(s) was similar to performing incision and curettage of chalazion, except that the site was above the tarsal plate and extended towards the fornix. Then insertion of aponeurosis was dissected away from the anterior tarsal surface, and the more superiorly located levator was plicated on it with double arm suture(s). No tissue was excised in this procedure. Surgical success was defined as a postoperative margin reflex distance $(M R D)>2 \mathrm{~mm}$ and $<4.5 \mathrm{~mm}$, interlid height $<1 \mathrm{~mm}$ and satisfactory contour. Results Forty-four lids of 27 patients were included. Preoperative mean MRD was 0.48 $+/-0.56 \mathrm{~mm}$. Severe ptosis of MRD $<1 \mathrm{~mm}$ was present in $34 / 44$ patients $(77.3 \%)$. The postoperative mean MRD was $2.49+/-$ $0.53 \mathrm{~mm}$, and mean improvement was 2.02 $+/-0.61 \mathrm{~mm}$, which was statistically significant $(P<0.001)$. The overall success rate was $38 / 44(86.4 \%)$.

Conclusions Minimal incision posterior approach to levator plication was effective for the correction of aponeurotic ptosis with moderate to good levator function.

Eye (2015) 29, 483-491; doi:10.1038/eye.2014.318; published online 23 January 2015
\end{abstract}

\section{Introduction}

The popularity of posterior approach ptosis repair has waxed and waned over the years.
Nevertheless, a survey of members of the American Society of Ophthalmic Plastic and Reconstructive Surgery published in 2011 revealed that $74 \%$ of surgeons performed posterior approach ptosis surgery. ${ }^{1}$ Muller's muscle-conjunctival resection (MMCR), in particular, was found to attain better cosmetic results as compared with patients who underwent anterior approach levator advancement, and predictable outcomes without the need for intraoperative cooperation of the patient or adjustment. A comparative study of anterior levator advancement vs MMCR reported a $22 \%$ lower revision rate for MMCR and a statistically better outcome. ${ }^{2}$

On the basis of the theory that the aponeurosis is the main transmitter of contraction of the levator to tarsus, techniques for levator aponeurosis advancement through posterior approach have been previously described. ${ }^{3-7}$ These techniques avoid resection of conjunctiva which prevents dry eyes, and may be more effective in patients with negative response to phenylephrine test as well as correcting severe ptosis. Some surgeons may prefer intraoperative adjustment of lid height instead of using a preoperative algorithm to determine the amount of tissue resection.

Less invasive, yet effective, surgical techniques are of interest to both patients and surgeons. This study describes a technique of posterior approach to levator plication through minimal incision(s) near the fornix without resection of conjunctiva and Muller's muscle. No suture is required to close the small incision site(s). This study aims to evaluate the effectiveness of this technique for correction of adult involutional ptosis under local anesthesia which allows intraoperative adjustments of lid height and contour.

\section{Methods}

Surgical records from a single surgeon (DSN) were reviewed to identify all patients
Department of

Ophthalmology, Tung Wah Eastern Hospital, Hong Kong

Correspondence: DS Ng, Department of Ophthalmology, 9/F, Tung Wah Eastern Hospital, 19 Eastern Hospital Road, Causeway Bay, Hong Kong, Hong Kong

Tel: +852 21626901

E-mail: dr.dannyng@ gmail.com

Received: 15 September 2014

Accepted in revised form: 25 November 2014

Published online:

23 January 2015

The work has not been previously presented 
undergoing minimal incision posterior levator plication for ptosis repair between August 2013 and June 2014. Institutional Review Board approval was obtained for a retrospective chart review of these patients. Inclusion criteria included patients' age greater than 18 years, acquired aponeurotic ptosis, levator function (LF) equal or above $6 \mathrm{~mm}$ and surgery performed under local anesthesia without sedation. LF of less than $6 \mathrm{~mm}$, inadequate followup (less than 3 months postoperatively), required skin or fat blepharoplasty, those who underwent concurrent brow surgery, presence of a superior filtering bleb, previous history of trauma and ptosis of congenital, myogenic and neurogenic etiologies were excluded.

Preoperative data collected include age, sex, previous history of ptosis surgery and types, upper eyelid margin reflex distance (MRD), and LF. MRD is defined as the distance between the central corneal light reflection and the upper eyelid margin. Using the examiner's hand to fixate the brow, eyelid excursion from extreme downgaze to upgaze was measured and recorded as LF. Response to $2.5 \%$ phenylephrine instillation was also documented, with elevation of lid height $1 \mathrm{~mm}$ or more deemed a positive result.

Duration of surgery for each ptotic eyelid was collected. Patients were followed up within 1 week postoperatively. MRD and LF were measured in each follow-up visits. Measurements at the last follow-up visit were used for comparison. Surgical success was defined as postoperative MRD of $\geq 2 \mathrm{~mm}$ and $\leq 4.5 \mathrm{~mm}$, and an interlid height difference of $\leq 1 \mathrm{~mm}$. Any complications and recurrence of ptosis necessitating surgery were recorded. All patients had pre- and post-operative photographs taken, the latter being performed 1-3 months postoperatively. Statistical analysis, including Wilcoxon signed-rank test was conducted by JMP statistical software (V7.0 for Windows, SAS Institute Inc, Cary, NC, USA).

\section{Surgical technique}

Topical anesthetic (proparacaine, $0.5 \%$ solution) was applied to the eye $5 \mathrm{~min}$ before the procedure was performed. The skin crease was marked at a matching height to the fellow eye. If bilateral ptosis was present, the crease height was based on central tarsal height, which averages 6.5-8.0 mm (slightly lower in Asians). The involved upper eyelid was everted over a Desmarres retractor. To avoid distorting upper lid tissue and contour, a small amount of local anesthesia of $0.5-1 \mathrm{ml}$ of $2 \%$ lidocaine with 1:80000 adrenaline was injected into the subconjuctival layer at the start of the procedure. Patients remained fully alert throughout the procedure without sedation. If the patient complained of pain at any time during the operation, small bolus of local anesthetic was injected. Two 5-0 silk traction sutures were placed at the lateral and medial sides at the superior edge of tarsal plate to double evert the upper lid (Figure 1a and b). The free ends of tractions sutures were clamped to the drape when the upper lid fornix was adequately exposed. Figure $3 \mathrm{a}$ is a schematic diagram that illustrates the essential upper lid anatomy after double eversion. A monopolar diathermy (Erbe USA Incorporated, Marietta, GA, USA) was applied to the conjunctiva immediately above the superior tarsal border for a $3-5 \mathrm{~mm}$ vertical incision towards the fornix, similar to performing incision and curettage of a chalazion, except that the incision site was above the tarsal plate (Figure 1c). The incision depth was through the thin layer of Muller's muscle until the appearance of levator aponeurosis, which could be directly visualized as a whitish tissue layer, or identified by its movement when the patient looks up and down. A pair of Stevens scissors was used for blunt dissection to further undermine the conjunctiva and Muller's muscle layer from the posterior belly of the levator aponeurosis. At the superior edge of tarsus, sharp scissors entered the plane just superficial to the anterior surface of tarsal plate to dissect the insertion of aponeurosis away from the anterior tarsal surface until 2-3 mm below the superior tarsus edge (Figures $2 \mathrm{a}$ and $3 b)$. A double-armed 6-0 vicryl suture was placed through the posterior belly of the levator aponeurosis and passed through partial thickness of the tarsal plate at its anterior surface (Figure $2 b$ and $c$ and Figure $3 c$ ). The traction sutures were released, and then the sutures were externalized through skin at the level of lid crease. A knot was tied if the eyelid position was deemed to be satisfactory (Figure 2d). Figure 3d illustrates the location of the levator plication suture when the upper lid returns to its primary position. Lid height and contour were assessed with the patient seated upright (Figure 4b, Left). If the lid was too low after the first suture, a second suture was placed just next to the first suture, passing higher through the levator aponeurosis and again through the tarsal plate and skin to avoid the undue delay of removing the first suture. If the upper eyelid contour appeared peaked after the first suture, then this was relaxed and a second suture was placed. If the lid appeared low medially or laterally, additional sites for levator plication was performed by the same method accordingly until satisfactory lid contour was achieved. The incision(s) wound through the conjunctiva and Muller's muscle were left to heal by primary intention. The absorbable sutures were not removed and were left to dissolve spontaneously in the postoperative period.

\section{Results}

The minimal incision posterior approach for levator plication technique was performed on 44 lids of 27 patients. All patients were of Asian ethnicity. Twenty-five 
a

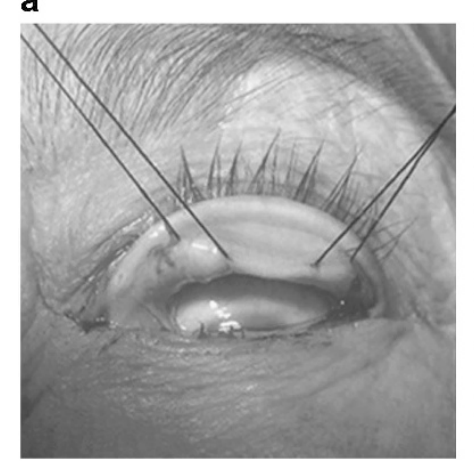

b

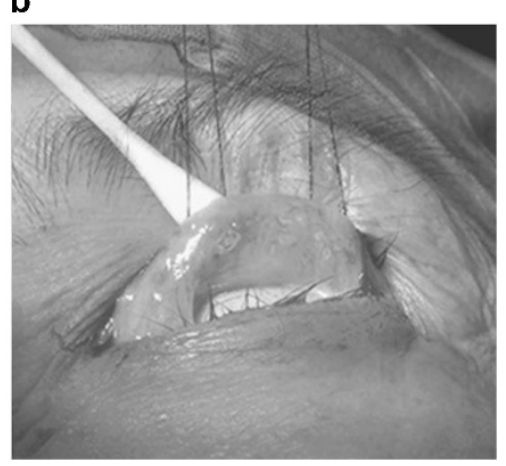

c

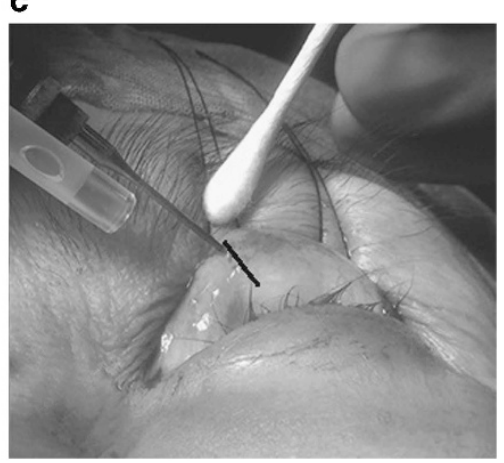

Figure 1 (a) Upon lid eversion, two 5-0 silk traction sutures were placed at the lateral and medial sides at the superior edge of tarsal plate. (b) The tarsal plate was flipped by traction sutures pulled towards the patient's forehead, aided by the tip of a cotton swap pushing the underside of tarsal plate. This maneuver double everted the upper lid and the fornix was adequately exposed. (c) Vertical incision of conjunctiva immediately above the superior tarsal border for $5 \mathrm{~mm}$ towards the fornix using a monopolar diathermy needle powered by an electrosurgical unit, similar to performing incision and curettage of a chalazion, except that the incision site was above the tarsal plate. A black line was drawn to outline the intended length of the vertical incision. A suction cannula was shown next to the needle.

of 27 patients (92.6\%) were Chinese, one patient was Indian, and another was Nepalese. Three lids of two patients had recurrent blepharoptosis with history of previous anterior approach levator advancement surgery. A total of 21 right and 23 left eyelids were included with 17 patients $(63 \%)$ undergoing bilateral procedures. Sixteen out of 17 bilateral cases were performed simultaneously. There were 10 male and 17 female. The mean age was $72.5+/$ - standard deviation (SD) 9.9 (range 52-95) years. Operated eyelids had LF ranging from 7 to $15 \mathrm{~mm}$ with mean of $11.5 \mathrm{~mm}+/-$ SD $1.9 \mathrm{~mm}$. The response rate to preoperative $2.5 \%$ phenylephrine test was $23 / 44(52.3 \%)$ and $11 / 44(25 \%)$ had negative response. The response was not documented in 10 patients $(22.7 \%)$.

All patients had a minimum of 3 months follow-up with a mean of $17.5+/-$ SD 6.3 (range 12-31) weeks. Preoperative MRD ranged from 0 to $2 \mathrm{~mm}$ with mean $0.48+/-$ SD $0.56 \mathrm{~mm}$. Severe ptosis with $\mathrm{MRD}<1 \mathrm{~mm}$ was present in $34 / 44$ patients $(77.3 \%)$. The postoperative mean MRD was $2.49+/-0.53 \mathrm{~mm}$, and mean improvement was $2.02+/-0.61 \mathrm{~mm}$, which was statistically significant $(P<0.001)$. The overall success rate was $38 / 44(86.4 \%)$. Eyelid symmetry to within $0.5 \mathrm{~mm}$ was achieved in $37 / 44(84.1 \%)$. In the subgroup with severe ptosis, the success rate was 29/34 (85.3\%). The success rate in phenylephrine-positive group was $21 / 23(91.3 \%)$, vs 8/11 (72.7\%) in the phenylephrinenegative group. All three lids with recurrent ptosis met the postoperative criteria for success. Figures 4 and 5 illustrate the pre- and post-surgical results for unilateral and bilateral ptosis correction.

Of the 44 lids operated, 4 received one suture, 26 received two sutures, 13 received three sutures, and 1 received four sutures. The mean duration of operation for each eyelid was $31.1+/-14.2$ (range 8-66) min. Most patients did not require extra bolus of local anesthesia injection after the start of the procedure, with the exception for those who had prolonged operation and for few cases with very low pain threshold.

All six unsuccessful lids of six patients were due to under-correction, and all of them were noted within postoperative 4 weeks. Five of them had severe ptosis of $\mathrm{MRD}<1 \mathrm{~mm}$ preoperatively, and four patients opted for reoperation, using the same minimal incision posterior approach levator plication technique as previous. All of them met the success criteria after reoperation. Seven patients $(15.9 \%)$ reported foreign body sensation which resolved within the first week after surgery with topical lubricating eye drops. No other complications were found during follow-up.

\section{Discussion}

We described a surgical technique for correction of involutional ptosis involving the plication of levator apneurosis by minimal incision via transconjunctival route. The overall success rate was $86.4 \%$ and all patients achieved a satisfactory contour. Using the same success criteria, other studies of posterior approach ptosis surgery also reported similar success rate of over $80 \% .^{5-8}$ McCulley et $a l^{8,9}$ described a large series of traditional anterior approach to levator advancement and reported $77 \%$ success, and a simplified anterior approach technique of using a single adjustable suture had success rate of $74 \%$. A study of 10 years' experience in MMCR in Asian unilateral ptosis reported $82.8 \%$ success defined by interlid MRD difference of $\leq 1 \mathrm{~mm} .{ }^{10}$ Nonetheless, direct comparison of outcomes with other published results in the literature is difficult because of different study 
a

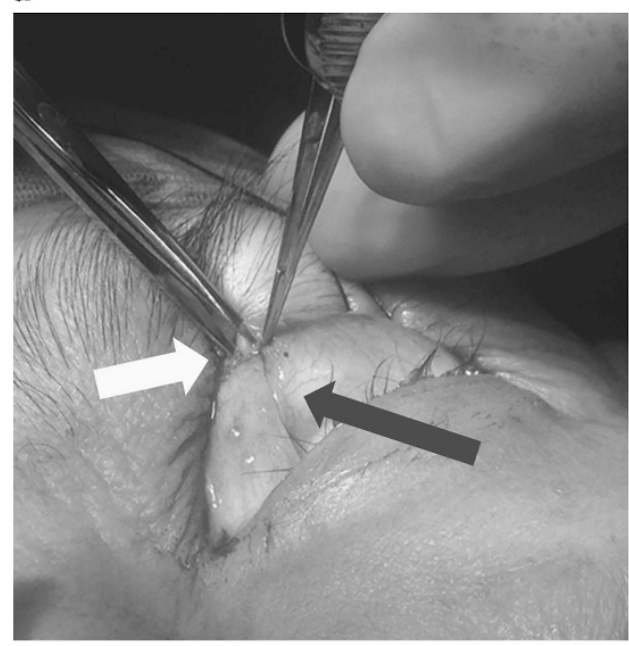

C

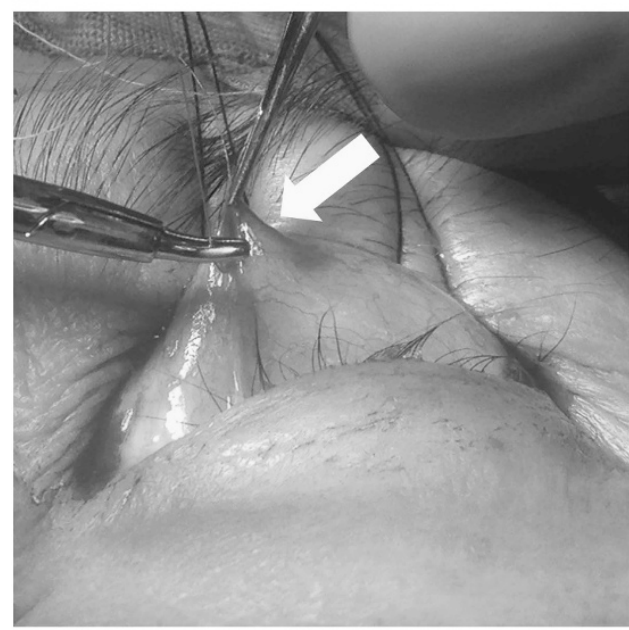

b

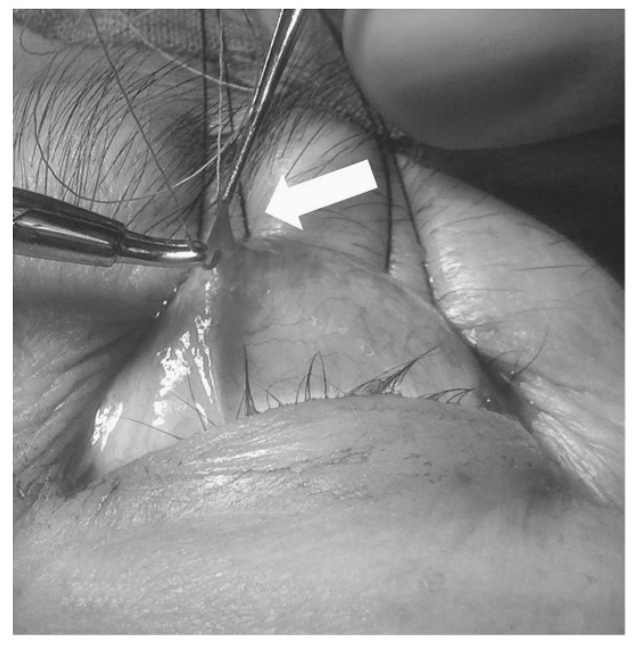

d

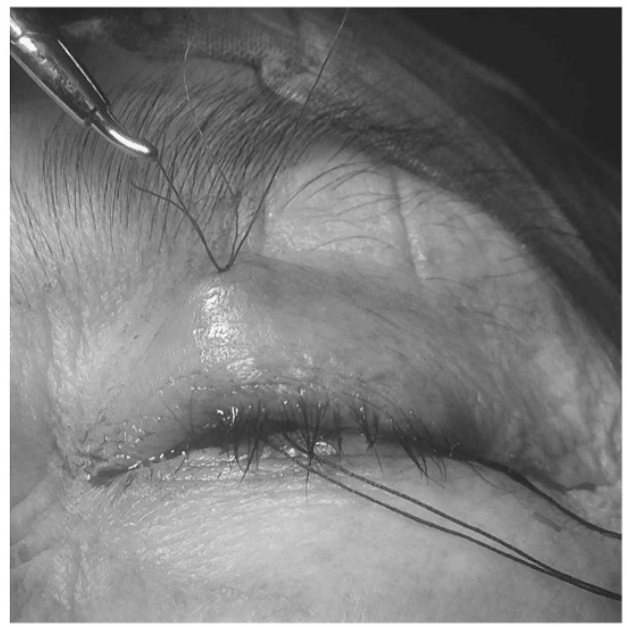

Figure 2 (a) A pair of Stevens scissors entered the plane just superficial to the anterior surface of tarsal plate to dissect the insertion of the aponeurosis away from the anterior tarsal surface until $2-3 \mathrm{~mm}$ below the superior tarsus edge. White arrow indicates the anterior surface of tarsus plate. Black arrow indicates the prior vertical incision through conjunctiva and Muller's muscle layers. The levator aponeurosis could be directly visualized as a whitish tissue layer, or identified by its movement when the patient looks up and down. (b) A double-armed 6-0 vicryl suture was placed through the posterior belly of the levator aponeurosis indicated by white arrow. (c) The sutures were then passed through partial thickness of the tarsal plate at its anterior surface. White arrow indicates the location of levator aponeurosis plication. (d) The sutures were externalized through skin at the level of the lid crease. Lid height and contour were assessed in primary position. The sutures were tied if the eyelid position was deemed to be satisfactory.

design, patient demographics, and definition of success. ${ }^{11,12}$

The average time needed to operate for each eyelid was $31.1 \mathrm{~min}$, which was comparable with the average time of $26.3 \mathrm{~min}$ reported by Frueh et al ${ }^{13}$ using the small incision, minimal dissection anterior approach procedure. In contrast, the same author reported an average time of $56.6 \mathrm{~min}$ for traditional lid crease incision approach. ${ }^{13}$

None of the cases in this series were complicated by overcorrection. In a 2-year prospective audit of ptosis surgery in Singapore, $10.1 \%$ of eyelids required readjustment surgery. ${ }^{11}$ We report a similar rate
(4/44 eyelids, $9.1 \%$ ) of reoperation in this study and all of these were due to undercorrection, of which $5 / 6$ patients had severe ptosis of MRD $<1 \mathrm{~mm}$. In McCulley et al's large series of 828 patients, severe blepharoptosis, demonstrated by MRD and LF measurements, were at increased risk of undercorrection.

Our minimal incision technique differs from previously described posterior approach ptosis surgeries which involved advancement or resection of levator aponeurosis because we avoided violation of the orbital septum. ${ }^{3-7}$ Collin's technique of levator aponeurosis reinsertion involved exposing the anterior surface of levator by excising $2 \mathrm{~mm}$ of the superior tarsus and 
Muller's muscle, followed by dissection of the orbital septum and retraction of the preaponeurotic fat pad to convert a posterior approach to the familiar anatomical view of an anterior approach ptosis repair. ${ }^{3}$

Ichinose et $^{\mathrm{al}} \mathrm{l}^{4}$ reported a technique for posterior approach to levator advancement via incision of conjunctiva and Muller's muscle horizontally along medial 2/3 of the superior border of tarsus. Similar to Collin's approach, their technique required incision of the orbital septum to expose the anterior surface of the levator before advancing it to the fronto-superior part of tarsal plate. Muller's muscle was reattached by sutures to

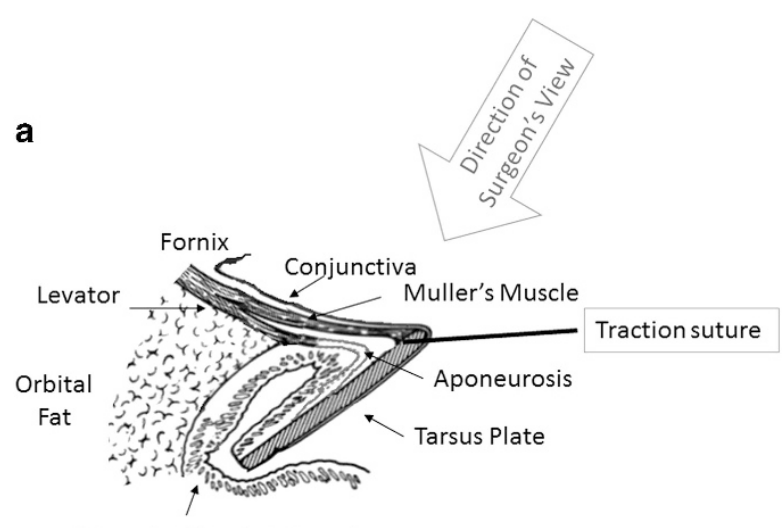

Skin and Orbicularis Muscle

b

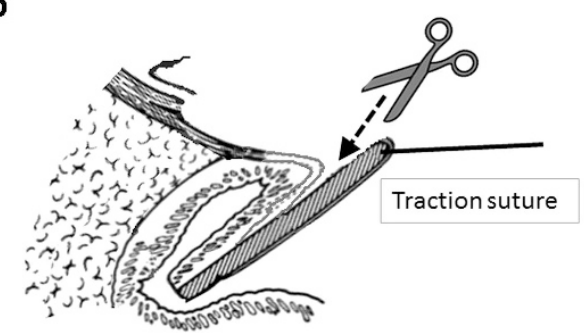

C

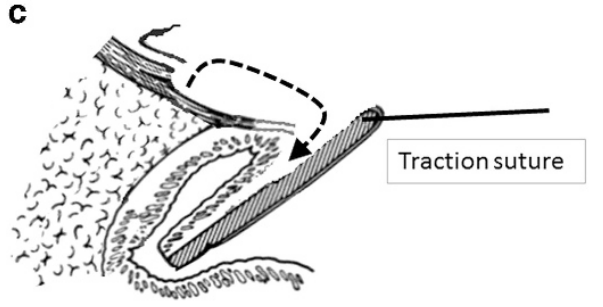

d

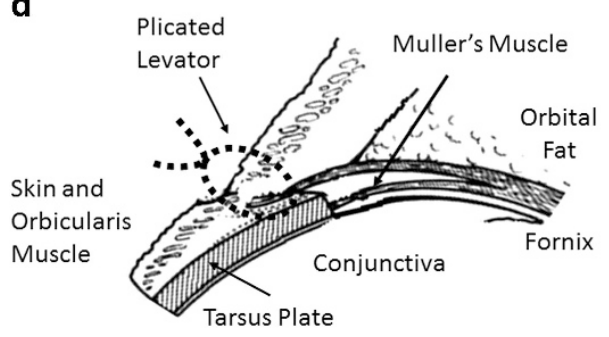

the superior edge of tarsal plate. The author noted longer postoperative period required for eyelid to reach its highest desired position compared with anterior approach for aponeurosis repair without dissection of Muller's muscle. Hence, the lateral 1/3 of Muller's muscle was spared to keep its nervous innervation intact in order to retain some function. ${ }^{14,15}$

Patel et al and Malhotra et al ${ }^{5,6}$ reported their posterior approach to aponeurosis 'white line' advancement without resecting Muller's muscle and conjunctiva while preserving the integrity of the orbital septum in adults under local and general anesthesia, respectively. Al-Abbadi et $a l^{7}$ described a similar technique on congenital ptosis patients. This technique involves incision of conjunctiva and Muller's muscle horizontally along the length of superior border of tarsus, and then to dissect it off as a composite flap until the appearance of the 'white line' which represented the posterior border of levator aponeurosis. The 'white line' was advanced to the conjunctival surface of the tarsal plate, $1 \mathrm{~mm}$ below its superior border. Al-Abbadi et $a^{7}$ have modified this technique for correction of more severe congenital ptosis by dissecting a larger composite flap for better exposure to plicate the levator beyond the aponeurosis. The principal goal of the 'white line' advancement technique was to identify and advance the lower border and posterior surface of the aponeurosis without breaching the orbital septum. Our technique differed in that double eyelid eversion allowed the advanced levator aponeurosis to reinsert at the anterior surface of tarsal plate to restore its original anatomical position. Double eversion of the upper lid with the aid of traction sutures, maybe easier to perform in Asian eyelids because of reduced tarsal height $(6.5-8 \mathrm{~mm})$ when compared with Caucasian eyelids. ${ }^{16}$ Our success rate was $86.4 \%$, which

Figure 3 Schematic diagram of the minimal incision posterior approach levator plication surgery. (a) Illustration of the essential anatomical components of the upper eyelid after double eversion aided by 5-0 silk traction sutures placed at the superior border of tarsus plate. (b) Conjunctiva and Muller's muscle were vertically incised. (These two layers were removed from the schematic diagram for illustration purpose.) As no tissue was resected when performing the procedure, bleeding was easily controlled, which facilitated the identification of levator aponeurosis. At the superior edge of tarsus, sharp scissors entered the plane just superficial to the anterior surface of tarsal plate to dissect the insertion of aponeurosis away from the anterior tarsal surface until $2-3 \mathrm{~mm}$ below the superior tarsus edge (black dotted arrow). (c) A double-armed 6-0 vicryl suture was placed through the posterior belly of the levator aponeurosis and passed through partial thickness of the tarsal plate at its anterior surface (black dotted arrow). (d) Upper eyelid and the final location of the levator plication sutures (black dotted line) illustrated in the primary position. The knot was externalized to skin lid crease. 
a
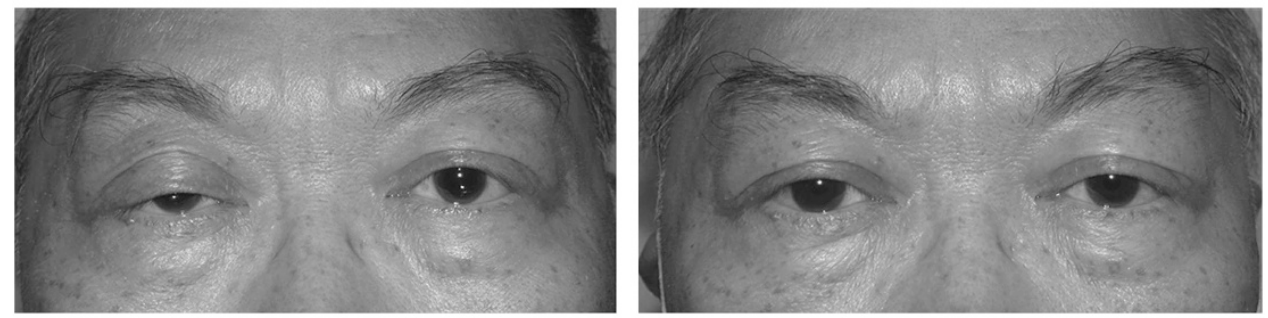

b
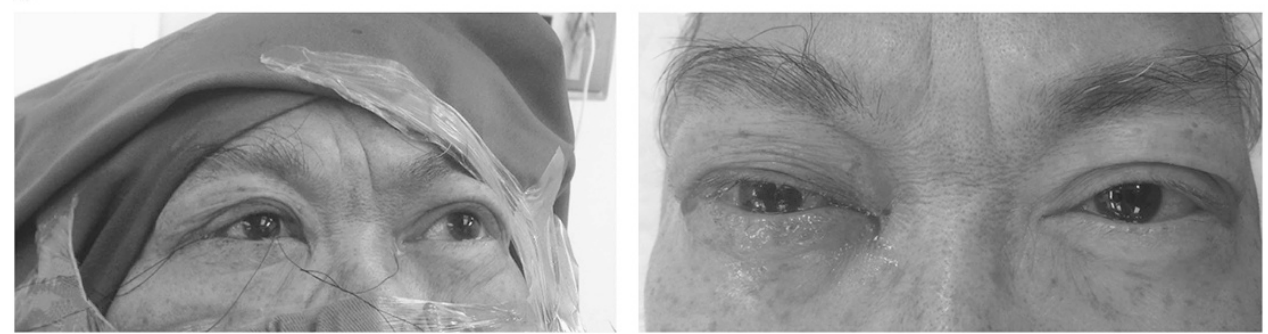

Figure 4 (a) Left: A 67 year-old man with severe right unilateral involutional ptosis with preoperative MRD $0 \mathrm{~mm}$. Right: Surgical result 1 month postoperatively. (b) Left: The same patient seated upright during intraoperative right upper lid height adjustment to match the height and contour with the fellow eye. Right: Day 1 post operation, notice the right upper lid has almost achieved the desired height and contour with minimal ecchymosis.
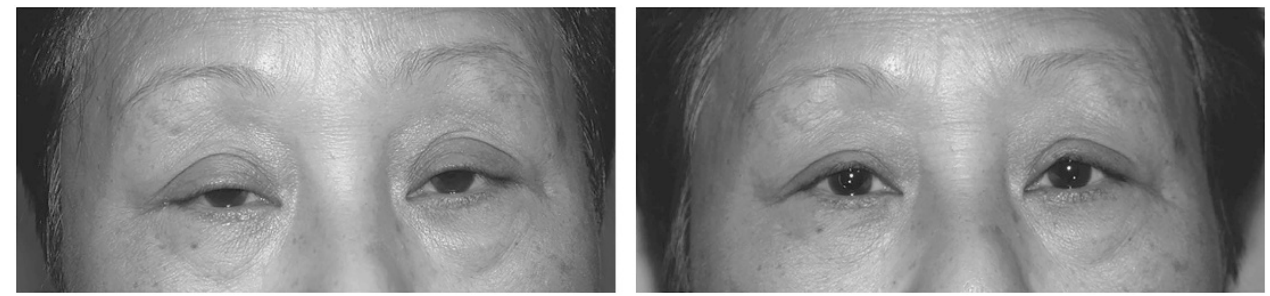

Figure 5 (Left) A 66-year-old woman with bilateral involutional ptosis with preoperative right eye MRD $0 \mathrm{~mm}$ and left eye $0.5 \mathrm{~mm}$. (Right) Surgical result 1 month postoperatively.

is comparable with the success rate of $87.3 \%$ reported by Patel et $a l^{5}$ using the same criteria.

Internal ptosis surgeries that involve posterior lamellar structures including the tarsus, conjunctiva, and Muller's muscle without surgical resection, reinsertion, or advancement of the levator aponeurosis have been effective. In 1975, Putterman and Urist ${ }^{17}$ described the MMCR technique with a special clamp to dissect the Muller's muscle together with conjunctiva off its bed on the aponeurosis. Reports of MMCR suggested a highly successful rate in ptosis correction ranging from 85 to $90 \% .{ }^{18,19}$ However, the majority of successful cases that underwent MMCR had mild ptosis with good to moderate LF. The only comparative study of anterior approach levator advancement vs MMCR revealed that patients who underwent anterior levator advancement had significantly lower preoperative MRD but were able to attain similar postoperative outcomes as the MMCR group. ${ }^{2}$ The result from this study implied that advancement of the levator aponeurosis with intraoperative adjustment of eyelid height is a more powerful surgical procedure than MMCR that could be performed in any degree of involutional ptosis with adequate LF. The aponeurosis was thought to be the main transmitter of contraction of the levator to the tarsus, and to ignore the aponeurotic defect was considered an illogical solution for correcting involutional ptosis. ${ }^{20}$ Nonetheless, our understanding of the anatomical reason for success of MMCR has evolved. Dresner ${ }^{18}$ proposed that excision of posterior lamellar had the effect of plicating the levator aponeurosis from behind. This was further supported by compelling evidence from cadaveric study conducted by Marcet et $a l^{21}$ which demonstrated conjunctiva and Muller's muscle in all resection specimens, and the levator aponeurosis was plicated in the cadavers. Resection of the Muller's muscle wound transmit the contraction force of the levator muscle directly to the tarsal plate instead of through its aponeurotic attachment.

Nevertheless, MMCR has lower success rate in patients 
with severe ptosis, either because direct surgical reinsertion and advancement of the levator aponeurosis is more effective, or the amount of tissue that was blindly captured with the ptosis clamp during MMCR was insufficient. In our study, a large proportion of patients (77.3\%) had severe ptosis, and the $85.3 \%$ success rate was achieved by our minimal incision posterior levator plication technique for this subgroup.

The predictability and result of MMCR also depends on a positive response to preoperative phenylephrine test. A variety of preoperative assessment algorithm for determining the amount of tissue resection have been proposed to optimize the surgical results for similar degrees of ptosis. ${ }^{18,22,23}$ Varying responses to phenylephrine may have been caused by different severity of fatty degeneration of Muller's muscle and viability of its adrenergic receptors. ${ }^{24}$ Although it would have been useful to compare the phenylephrine response-positive with the response-negative group, the sample size in this study was too small for statistically significant result. However, the principle of our technique was to allow direct visualization of the eyelid anatomy with subsequent intraoperative lid height and contour adjustments. This was expected to be effective irrespective of the response to phenylephrine, and omitted the need for preoperative algorithms to calculate the amount of resection as required in the closed clamp technique that was originally described by Putterman et al. ${ }^{17}$ Despite its irrelevance to the surgical success of posterior approach levator repair or to the open sky MMCR technique, preoperative phenylephrine test can still be useful in demonstrating the potential postoperative outcomes to patients and to unmask contralateral latent ptosis by way of Hering's law.

An open sky technique of mullerectomy was described which allowed direct visibility of relevant eyelid structures for intraoperative adjustment by placement of sutures higher up in the residual stump of Muller's muscle. ${ }^{24-27}$ Peter et al, ${ }^{24}$ in a large retrospective cohort of over 300 patients who were followed from 4-47 months, reported over $90 \%$ of cases met the success criteria. Most of the eyelids $(96 \%)$ with severe ptosis of MRD $<1 \mathrm{~mm}$ also achieved target lid height. This technique was also found to be effective in phenylephrine-negative patients, because its success did not rely on the amount of Muller's muscle resected but rather from the consequent advancement of the levator. ${ }^{24,26,27}$ However, $6 \%$ required augmentation of a subtotal Muller resection by either a small tarsectomy or conversion to posterior approach levator resection as described by Collin. ${ }^{3,24}$ None of the eyelids in our study required augmentation surgery. In severe blepharoptosis, the vertical incisions could be extended towards the fornix to allow plication of the levator that was located superior to the aponeurosis without the need to dissect a large conjunctiva and Muller's muscle flap. These cases may require higher number of double-arm sutures (usually 3-4) to plicate the levator to achieve a satisfying upper lid contour. If undercorrection was noticed in the early postoperative period, reoperation using the same technique but placing more sutures and aiming for slight intraoperative lid height overcorrection yielded successful results. Peter et $a l^{24}$ reported $92 \%$ of patients felt that their initial postoperative lid height was maintained over the long-term, in contrast to anterior aponeurosis surgery in which lid height tends to drop over time. The outcome of our study in the early postoperative period is encouraging, and further long-term observation is required to evaluate whether the lid height improvement could be maintained over time.

Proponents of anterior approach to ptosis repair have raised concern regarding to excision of tarsus and conjunctiva that resulted in damaging the goblet cells for mucin secretion, accessory lacrimal gland and meibomian glands that were essential for the stability of the 3-layer tear film. ${ }^{28}$ Wee et $a l^{29}$ reported dry eye-related symptoms after MMCR with significant differences in Schirmer test and ocular surface disease index score before and after surgery. Although there is insufficient evidence on the aftermath of conjunctival excision, it is still desirable to conserve healthy conjunctival tissue to minimize the risk of dry eye symptoms and to prevent shallowing of the upper fornix, which is also of concern to anophthalmic patients who needed ptosis correction. ${ }^{27}$ Recent techniques of posterior approach ptosis repair were modified to prevent excising any conjunctival tissue. ${ }^{4-7,24,27}$ Regarding to the open sky mullectomy, Peter et al ${ }^{24}$ reported that $4 \%$ of eyelids developed complications related to postoperative surface drying and presence of sutures on the palpebral conjunctiva, including corneal abrasions, suture abscesses, prolonged postoperative pain, and blood cyst. We have not encountered any of these complications because our minimal incision technique does not require placing suture on the palpebral conjunctiva. However, we also acknowledge that the sample size of this study may be under-powered to detect the risk of these postoperative complications.

Limitations of the current study stem from its retrospective design. Longer follow-up period could reveal whether the outcome of this technique is durable. Prospective comparison studies between different surgical approaches could determine which is more effective in treating patients with severe ptosis and in those who have negative response to phenylephrine. Furthermore, a study of Schirmer test and tear breakup time in postoperative patients can reveal whether it is beneficial to preserve conjunctival tissue. 
This study has demonstrated that minimal incision posterior approach levator plication is an effective and safe technique in correction of involutional ptosis with good to moderate LF, including those with severe ptosis $(\mathrm{MRD}<1 \mathrm{~mm})$ and negative response to phenylephrine test. The major advantage is not requiring the placement of suture material in the palpebral conjunctiva. In addition to preservation of conjunctival tissue, our posterior approach is able to advance and reinsert the levator aponeurosis without extensive tissue dissection. This could be favorable for intraoperative hemostasis, reduction of operation time and the amount of local anesthesia injection, and early resolution of postoperative ecchymosis. Previous studies on levator aponeurosis repair techniques have noted difficulty in intraoperative lid height adjustment because of temporary compromised LF by local anesthesia. ${ }^{30}$ Figure $4 \mathrm{~b}$ illustrates a patient during intraoperative lid height adjustment (left) and his appearance on postoperative day 1 (right). Patients in our study noticed that ecchymosis soon resolved and eyelids were lifted to the desired height shortly after operation. This technique is not recommended for patients who needed concurrent skin or fat blepharoplasty, or those with poor LF.

\section{Summary}

What was known before

- The conventional Muller's muscle conjunctival resection technique is more effective and predictable than anterior approach levator advancement in patients with mild ptosis with reported success rates over $80 \%$.

- However, surgical results from this technique relies on preoperative algorithm according to the lid's response to phenylephrine eye drops. Also, it is concerned that resection of conjunctiva will damage goblet cells and accessory lacrimal glands leading to unstable tear film.

- An 'open-sky' Muller resection technique and a posterior approach 'white line' advancement technique have been described, which allow intraoperative adjustment of lid height and preserve conjunctival tissue.

What this study adds

- Minimal incision posterior approach levator plication is an effective and safe technique in patients with involutional ptosis, including those with severe ptosis of MRD $<1 \mathrm{~mm}$ and negative response to phenylephrine test.

- No suture is placed at the palpebral conjunctiva for wound closure and no conjunctiva is excised which prevent dry eyes and stitch-related complications.

- Double eyelid eversion allows surgical advancement and reinsertion of levator aponeurosis at the anterior tarsal surface without violating the orbital septum.

- Minimal incision technique reduces the amount of tissue trauma, bleeding, local anesthesia injection, and operation time, which facilitates intraoperative lid height adjustment and early postoperative recovery.

\section{Conflict of interest}

The authors declare no conflict of interest.

\section{References}

1 Aakalu VK, Setabutr P. Current ptosis management: a national survey of ASOPRS members. Ophthal Plast Reconstr Surg 2011; 27(4): 270-276.

2 Ben Simon GJ, Lee S, Schwarcz RM, McCann JD, Goldberg RA. External levator advancement vs Muller's muscleconjunctival resection for correction of upper eyelid involutional ptosis. Am J Ophthalmol 2005; 140(3): 426-432.

3 Collin JR. A ptosis repair of aponeurotic defects by the posterior approach. Br J Ophthalmol 1979; 63(8): 586-590.

4 Ichinose A, Tahara S. Transconjunctival levator aponeurotic repair without resection of Muller's muscle. Aesthetic Plast Surg 2007; 31(3): 279-284.

5 Patel V, Salam A, Malhotra R. Posterior approach white line advancement ptosis repair: the evolving posterior approach to ptosis surgery. Br J Ophthalmol 2010; 94(11): 1513-1518.

6 Malhotra R, Salam A. Outcomes of adult aponeurotic ptosis repair under general anaesthesia by a posterior approach white-line levator advancement. Orbit 2012; 31(1): 7-12.

7 Al-Abbadi Z, Sagili S, Malhotra R. Outcomes of posteriorapproach 'levatorpexy' in congenital ptosis repair. $\mathrm{Br} \mathrm{J}$ Ophthalmol 2014; 98(12): 1686-1690.

8 McCulley TJ, Kersten RC, Kulwin DR, Feuer WJ. Outcome and influencing factors of external levator palpebrae superioris aponeurosis advancement for blepharoptosis. Ophthal Plast Reconstr Surg 2003; 19(5): 388-393.

9 Meltzer MA, Elahi E, Taupeka P, Flores E. A simplified technique of ptosis repair using a single adjustable suture. Ophthalmology 2001; 108(10): 1889-1892.

10 Jang SY, Chin S, Jang JW. Ten years' experience with unilateral conjunctival mullerectomy in the Asian eyelid. Plast Reconstr Surg 2014; 133(4): 879-886.

11 Young SM, Lim LH, Seah LL, Choo CT, Chee EW, Shen SY et al. Prospective audit of ptosis surgery at the Singapore National Eye Centre: two-year results. Ophthal Plast Reconstr Surg 2013; 29(6): 446-453.

12 Scoppettuolo E, Chadha V, Bunce C, Olver JM, Wright M. Bopss. British Oculoplastic Surgery Society (BOPSS) National Ptosis Survey. Br J Ophthalmol 2008; 92(8): 1134-1138.

13 Frueh BR, Musch DC, McDonald HM. Efficacy and efficiency of a small-incision, minimal dissection procedure versus a traditional approach for correcting aponeurotic ptosis. Ophthalmology 2004; 111(12): 2158-2163.

14 Matsuo K. Restoration of involuntary tonic contraction of the levator muscle in patients with aponeurotic blepharoptosis or Horner syndrome by aponeurotic advancement using the orbital septum. Scand J Plast Reconstr Surg Hand Surg 2003; 37(2): 81-89.

15 Sisler HA. Surgical correction of blepharoptosis and lowereyelid retraction in dry-eyed patients. Arch Ophthalmol 1982; 100(5): 800-801.

16 Chen WP. Asian blepharoplasty. Update on anatomy and techniques. Ophthal Plast Reconstr Surg 1987; 3(3): 135-140.

17 Putterman AM, Urist MJ. Muller muscle-conjunctiva resection. Technique for treatment of blepharoptosis. Arch Ophthalmol 1975; 93(8): 619-623. 
18 Dresner SC. Further modifications of the Muller's muscleconjunctival resection procedure for blepharoptosis. Ophthal Plast Reconstr Surg 1991; 7(2): 114-122.

19 Putterman AM, Fett DR. Müller's muscle in the treatment of upper eyelid ptosis: a ten-year study. Ophthalmic Surg 1986; 17(6): 354-360.

20 Dortzbach RK, Sutula FC. Involutional blepharoptosis. A histopathological study. Arch Ophthalmol 1980; 98(11): 2045-2049.

21 Marcet MM, Meyer DR, Greenwald MJ, Roth S, Selva D. Proximal tarsal attachments of the levator aponeurosis: implications for blepharoptosis repair. Ophthalmology 2013; 120(9): 1924-1929.

22 Weinstein GS, Buerger Jr., GF. Modification of the Muller's muscle-conjunctival resection operation for blepharoptosis. Am J Ophthalmol 1982; 93(5): 647-651.

23 Perry JD, Kadakia A, Foster JA. A new algorithm for ptosis repair using conjunctival Mullerectomy with or without tarsectomy. Ophthal Plast Reconstr Surg 2002; 18(6): 426-429.

24 Peter NM, Khooshabeh R. Open-sky isolated subtotal Muller's muscle resection for ptosis surgery: a review of over 300 cases and assessment of long-term outcome. Eye 2013; 27(4): 519-524.

25 Lake S, Mohammad-Ali FH, Khooshabeh R. Open sky Muller's muscle-conjunctiva resection for ptosis surgery. Eye 2003; 17(9): 1008-1012.

26 Baldwin HC, Bhagey J, Khooshabeh R. Open sky Muller muscle-conjunctival resection in phenylephrine testnegative blepharoptosis patients. Ophthal Plast Reconstr Surg 2005; 21(4): 276-280.

27 Khooshabeh R, Baldwin HC. Isolated Muller's muscle resection for the correction of blepharoptosis. Eye 2008; 22(2): 267-272.

28 Anderson RL. Predictable ptosis procedures: do not go to the dark side. Ophthal Plast Reconstr Surg 2012; 28(4): 239-241.

29 Wee SW, Lee JK. Clinical outcomes of conjunctiva-Muller muscle resection: association with phenylephrine test-negative blepharoptosis and dry eye syndrome. J Craniofac Surg 2014; 25(3): 898-901.

30 Lucarelli MJ, Lemke BN. Small incision external levator repair: technique and early results. Am J Ophthalmol 1999; 127(6): 637-644. 